\title{
Derniers résultats sur le boson de Higgs au LHC
}

Lucia di Ciaccio ${ }^{(1)}$ (lucia.di.ciaccio@cern.ch)

et Gautier Hamel de Monchenault ${ }^{(2)}$ (gautier.hamel.de.monchenault@cern.ch)

(1) Laboratoire d’Annecy-le-Vieux de Physique des Particules, CNRS et Université de Savoie,

BP 110, 74941 Annecy-le-Vieux Cedex. Membre de l'expérience ATLAS.

(2) Service de Physique des Particules, Institut de Recherche sur les lois Fondamentales de l'Univers, CEA/Saclay,

91191 Gif-sur-Yvette Cedex. Membre de l'expérience CMS.

La découverte du boson de

Higgs, annoncée le 4 juillet 2012

au CERN par les collaborations

internationales ATLAS et CMS,

représente l'accomplissement

d'une quête dont l'origine

remonte aux années 1960.

Depuis cet événement,

les physiciens d'ATLAS et

CMS poursuivent l'étude

des propriétés de cette

nouvelle particule

fondamentale avec l'analyse

des données de collisions

de protons produites au Large

Hadron Collider (LHC)

du CERN en 2011 et 2012.

Quelques mois après

le redémarrage du $\mathrm{LHC}$

à l'issue de 18 mois de travaux

de consolidation, cet article

dresse le bilan des

connaissances expérimentales sur le boson de Higgs.

\section{Le rôle du boson de Higgs dans le modèle standard des particules}

Le siècle dernier a vu l'avènement du modèle standard (MS) de la physique des particules, une théorie quantique et relativiste dans laquelle une particule élémentaire est la représentation d'un champ fonction des coordonnées d'espace-temps [1].

Dans cette théorie, les propriétés intrinsèques d'une particule sont invariantes lorsqu'on soumet le champ qui lui est associé à certaines opérations de symétrie mélangeant ses différentes composantes. Les forces (ou interactions) fondamentales émergent lorsqu'on impose à l'invariance d'être locale, c'est-à-dire vérifiée lorsque ces opérations de symétrie, dites alors transformations de jauge, sont également des fonctions des coordonnées d'espace-temps. On donne le nom d'invariance de jange à cette propriété de symétrie locale, et l'ensemble des transformations de jauge forme le groupe de jauge de la théorie.

Pour satisfaire l'invariance de jauge, on introduit des bosons vecteurs (particules de spin 1), associés aux générateurs du groupe de jauge et médiateurs des interactions entre particules. Ainsi, le photon, les bosons W et $Z$, et les huit gluons, émergent par ce principe élégant de symétrie comme les médiateurs des interactions électromagnétique, nucléaire faible et nucléaire forte. La théorie englobe les interactions électromagnétique et nucléaire faible en une interaction unifiée, dite électrofaible.

Cependant, on se heurte à une difficulté. La renormalisabilité de la théorie, qui assure sa cohérence mathématique et son pouvoir prédictif, impose aux bosons vecteurs des interactions d'être de masse rigoureusement nulle. Or la force nucléaire faible, responsable en particulier de la radioactivité $\beta$, est une interaction à très courte portée $\left[{ }^{\star}\right]$ et, par conséquent, les bosons chargés $\mathrm{W}\left(\mathrm{W}^{+}\right.$et $\left.\mathrm{W}^{-}\right)$ et le boson neutre $\mathrm{Z}$ doivent être extrêmement massifs. C'est en effet le cas : les bosons W et $\mathrm{Z}$, mis en évidence expérimentalement en 1984 au CERN, ont une masse élevée, de l'ordre de 100 fois la masse du proton.

Le mécanisme de Brout, Englert et Higgs $(\mathrm{BEH})$, développé dans les années 1960, permet d'introduire la masse des bosons $\mathrm{W}$ et $\mathrm{Z}$ dans la théorie, tout en préservant son invariance de jauge et sa renormalisabilité. Ce mécanisme, dit de brisure spontanée de la symétrie de jauge électrofaible, met en œuvre un champ scalaire (c'est-à-dire de spin zéro), appelé champ de Higgs, et prédit l'existence de la particule à laquelle ce champ est associé, le boson de Higgs.

La masse des particules de matière, les quarks et les leptons chargés (électrons, positons, muons...), qui sont des fermions (particules de $\operatorname{spin} 1 / 2$ ), résulte également de leur interaction avec le champ de Higgs qui les " retarde » dans leur propagation dans le vide. Ces fermions acquièrent ainsi une inertie, et donc une masse, proportionnelle à l'intensité de cette interaction.

De nombreuses expériences ont contribué à la quête du boson de Higgs depuis les années 1970, finalement couronnée de succès en 2012 [2, 3]. Sa découverte par les expériences ATLAS et CMS au LHC établit la réalité physique du champ de Higgs et valide le mécanisme $\mathrm{BEH}$ pour engendrer la masse des particules élémentaires. Depuis la découverte, les études au LHC se concentrent sur l'analyse fine des propriétés du boson de Higgs, pour les confronter aux prédictions du MS.

Dans la suite de cet article, après une brève introduction sur les deux expériences ATLAS et CMS (voir encadré 1), nous présenterons les mesures relatives aux propriétés du boson de Higgs, qui s'appuient sur l'ensemble des données disponibles. 


\section{Le détecteur ATLAS}

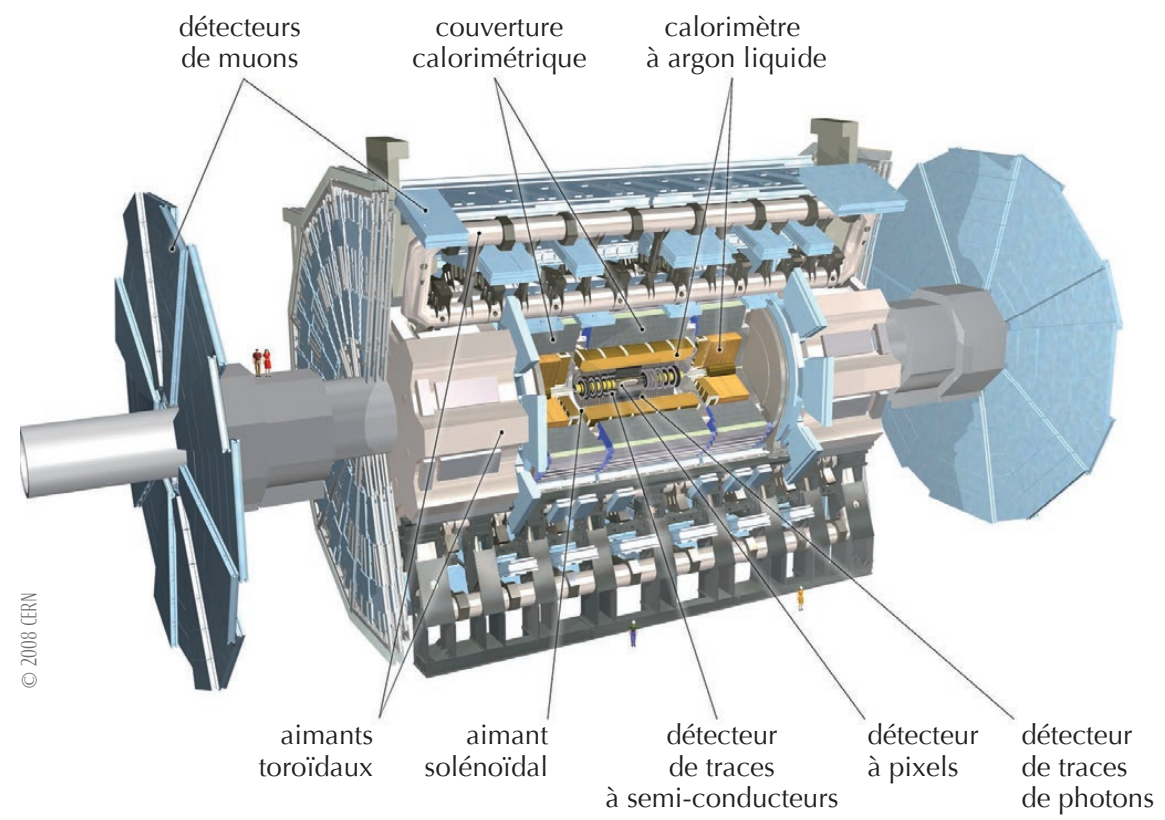

Le détecteur CMS

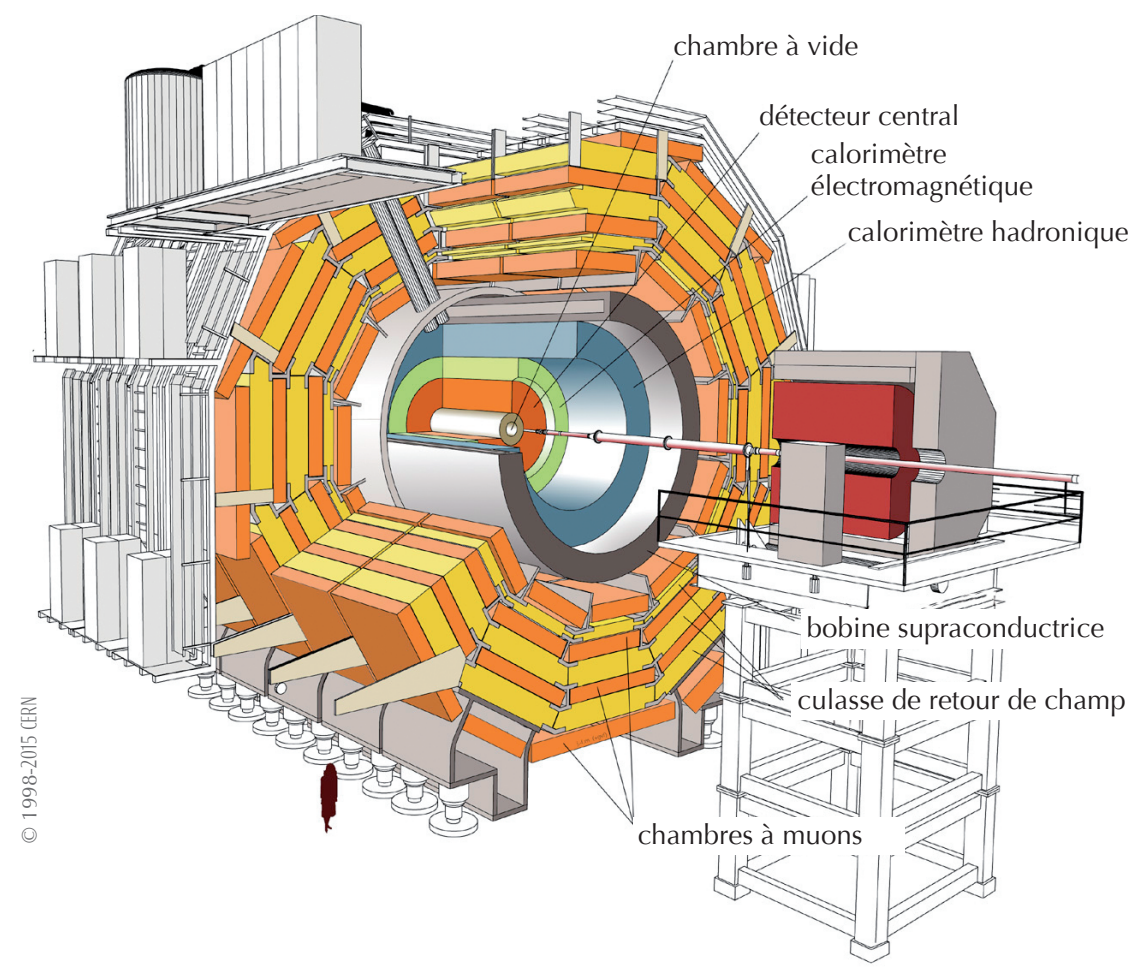

ATLAS et CMS désignent à la fois de grands détecteurs de physique des particules et les équipes de plusieurs centaines de physiciens et ingénieurs qui les ont conçus, construits, et en exploitent les données pour produire une quantité impressionnante de résultats de physique : depuis 2010, ATLAS et CMS ont publié chacune plus de 400 articles scientifiques dans des revues à comité de lecture.

Les détecteurs ATLAS et CMS sont conçus pour mesurer les énergies et impulsions des particules produites dans les collisions proton-proton. Les deux détecteurs diffèrent dans leur conception et leur configuration magnétique, mais présentent des performances comparables, ce qui permet la vérification indépendante des observations. ATLAS est un géant de 22 mètres de diamètre et 45 mètres de long, avec un système magnétique alliant un solénoïde supraconducteur central et trois grands toroïdes supraconducteurs à air pour la mesure des muons de haute énergie, une calorimétrie électromagnétique à argon liquide et une excellente couverture calorimétrique combinée. CMS, plus compact, s'articule autour d'un aimant supraconducteur solénoïdal de 4 mètres de diamètre et 16 mètres de longueur, assurant un champ magnétique homogène et intense (3,8 teslas) dans lequel sont plongés le détecteur de traces, le calorimètre électromagnétique à cristaux scintillants et le calorimètre hadronique, et dispose $d^{\prime}$ un système de reconnaissance des muons inséré dans le fer de la culasse de retour de champ.

Un événement désigne un croisement de deux paquets de protons, et présente selon les périodes entre 5 et 30 collisions protonproton réparties en moyenne sur une longueur d'environ 10 centimètres. La quantité de données pouvant être enregistrée est limitée, et ces collisions correspondent pour la plupart à des processus qui ne font pas l'objet principal d'investigation au LHC. Les systèmes de déclenchement permettent de réduire le taux d'événements de 10 mégahertz (la fréquence de croisement des paquets de protons) à quelques centaines de hertz et de sélectionner ainsi les processus (plus rares) que l'on recherche. Les quantités colossales de données sont reconstruites et analysées grâce à la grille informatique qui répartit la puissance de calcul dans les instituts et laboratoires participants autour de la planète [4]. 


\section{\〉 \\ La recherche du boson de Higgs avec ATLAS et CMS}

ATLAS et CMS sont deux des quatre grandes expériences qui analysent les collisions de protons (ou d'ions lourds pour ALICE) de très haute énergie délivrées par le LHC, le grand collisionneur de hadrons, un accélérateur de particules circulaire de 27 kilomètres de circonférence qui se situe au CERN, près de Genève.

ATLAS et CMS ont entamé leur campagne de prise des données en 2010 à une énergie de collision de $7 \mathrm{TeV}$ (environ 7000 fois l'énergie du proton au repos). À la fin de l'année 2011, les deux expériences ATLAS et CMS commençaient à détecter un frémissement dans la recherche du boson de Higgs, confirmé rapidement avec les premières données obtenues à $8 \mathrm{TeV}$ au printemps 2012.

Le 4 juillet 2012, lors d'une conférence conjointe organisée au CERN en présence de Peter Higgs et François Englert, prix Nobel de physique en 2013, les deux expériences annoncèrent indépendamment la découverte d'un nouveau boson de masse proche de $125 \mathrm{GeV}$ (133 fois la masse du proton, soit environ la masse de l'atome de xénon). Nous verrons que cette nouvelle particule a des propriétés compatibles avec celles du boson de Higgs prédit par le MS. C'est pourquoi nous l'appelons, peut-être abusivement, le boson de Higgs, étant conscients que d'autres particules du même type restent peut-être à découvrir.

Nous présentons ici des résultats obtenus par ATLAS et CMS avec l'ensemble des données accumulées à $7 \mathrm{TeV}$ en 2010 et 2011 et à $8 \mathrm{TeV}$ en 2012 [5]. Les données partielles analysées pour la découverte de juillet 2012 étaient en quantités comparables aux deux énergies. Les données aujourd'hui disponibles à $8 \mathrm{TeV}$ sont en quantité environ quatre fois supérieure.

La valeur de la masse du boson de Higgs n'est pas prédite mais, pour une masse donnée, la valeur de l'intensité de l'interaction (ou couplage) du boson de Higgs avec les autres particules élémentaires du MS est calculable. La théorie stipule que le couplage du boson de Higgs avec une particule est proportionnel à la masse ou à la masse au carré de celle-ci, selon qu'il s'agisse d'un fermion ou d'un boson. Cette propriété unique du boson de Higgs, liée au rôle du champ de Higgs dans le mécanisme responsable de la masse des fermions et des bosons, doit être vérifiée expérimentalement.

La conséquence de cette propriété est que le boson de Higgs n'interagit notablement qu'avec les particules les plus massives : le quark top, dont la masse est $173 \mathrm{GeV}$, les bosons W et $\mathrm{Z}$, de masses 80,5 et $91,2 \mathrm{GeV}$, et, dans une moindre mesure, le quark b (environ $4,5 \mathrm{GeV}$ ) et les leptons de troisième et deuxième génération, le lepton $\tau(1,8 \mathrm{GeV})$ et le muon $\mu(0,1 \mathrm{GeV})$, qui sont des répliques massives de l'électron $(0,0005 \mathrm{GeV})$.

La durée de vie du boson de Higgs est extrêmement courte, de l'ordre de $10^{-22} \mathrm{~s}$ : il parcourt une distance non mesurable, bien inférieure à la taille du proton, avant de se désintégrer. Pour reconstruire un boson de Higgs produit dans une collision proton-proton au LHC, il faut donc en

\section{Production et désintégrations du boson de Higgs} encadré 2

Le mode principal de production du boson de Higgs au LHC, responsable de $88 \%$ des bosons de Higgs produits, fait intervenir son couplage au quark top. C'est la production par fusion de gluons, notée ggF, ainsi appelée parce que le boson de Higgs émerge d'une boucle de quarks top virtuels, c'est-à-dire de masse très inférieure à leur masse nominale, issue de la collision de deux gluons provenant des protons incidents [6]. Les deux autres modes de production considérés ici font intervenir le couplage du boson de Higgs aux bosons vecteurs $\mathrm{W}$ et $\mathrm{Z}$ : ce sont la fusion de bosons vecteurs $(7 \%)$, notée $\mathrm{VBF}$, et la production associée avec un boson vecteur $(4 \%)$, notée $\mathrm{VH}$. Ces derniers modes de production sont essentiels pour extraire le signal de boson de Higgs pour les désintégrations où les bruits de fond sont abondants.

Malgré sa masse relativement faible, le boson de Higgs peut se désintégrer par l'intermédiaire d'une paire de bosons vecteurs W ou Z, à condition que l'un au moins des deux bosons soit virtuel [6]. Les bosons $W$ et $Z$ sont détectés dans leurs désintégrations en leptons, où lepton désigne ici soit un électron soit un muon. Le $W$ se désintègre en un lepton et un neutrino, lequel n'est pas détectable, et le Z en deux leptons de charges électriques opposées. En tenant compte de toutes les probabilités, la désintégration du boson de Higgs en deux leptons et deux neutrinos (WW) représente 1,1\% des cas, et en quatre leptons (ZZ), $0,028 \%$ des cas. Le mode $Z Z$, bien que très rare, est important car les quatre leptons dans l'état final sont détectés et leurs énergies mesurées précisément. Par conséquent, l'utilisation de ce mode permet une discrimination plus aisée du signal par rapport aux bruits de fond et une mesure précise de la masse du boson de Higgs.

Le boson de Higgs peut aussi se désintégrer en une paire de photons par le truchement de boucles de quarks top et de bosons W virtuels. Cette désintégration, notée $\gamma \gamma$, est également très rare $(0,23 \%)$, mais c'est l'une des plus importantes expérimentalement car les deux photons issus de la désintégration sont détectés et leurs énergies mesurées précisément.

La désintégration en une paire de quarks b $(57,7 \%)$ est délicate à étudier expérimentalement, car la production abondante de quarks b par paires au LHC constitue un bruit de fond colossal. Une façon d'extraire un signal consiste à s'intéresser à la production $\mathrm{VH}$, où l'on détecte le boson vecteur dans sa désintégration en leptons.

Enfin, le boson de Higgs se désintègre en une paire de leptons $\tau$ avec une probabilité de 6,3\%. Cette désintégration est également difficile à détecter car les leptons $\tau$, qui se désintègrent aussitôt en plusieurs particules dont au moins un neutrino, sont difficiles à identifier et à extraire des bruits de fond. Pour détecter un signal dans ce mode de désintégration, on exploite en particulier la production VBF. 
Les deux expériences ATLAS et CMS déploient des techniques d'analyse sophistiquées et un traitement statistique approprié pour extraire des signaux extrêmement faibles d'une quantité astronomique de données. La théorie prévoit en effet qu'un boson de Higgs est produit en moyenne tous les 65 millions de collisions, et seule une infime partie des bosons produits est détectable. Les données correspondent environ à un million de milliards de collisions proton-proton.

\section{Mesure des couplages et de la masse}

ATLAS et CMS observent indépendamment les trois modes de désintégration WW, ZZ et $\gamma \gamma$ du boson de Higgs. En exploitant la topologie des événements, les expériences estiment également les proportions des trois modes de production $\mathrm{ggF}$, $\mathrm{VBF}$ et $\mathrm{VH}$ [voir encadré 2].

La production de bosons de Higgs est relativement abondante dans le mode WW, mais la mesure de masse n'est pas précise en raison de la présence des deux neutrinos dans l'état final et de bruits de fond abondants. Ce mode est important pour la mesure du taux de production du boson de Higgs et la détermination de son spin. À l'inverse, la production de bosons de Higgs dans le mode ZZ est faible, quelques dizaines d'événements sélectionnés par ATLAS et CMS, mais le bruit de fond est modéré et la mesure de la masse très précise (fig. 1). D'autre part, le mode ZZ permet de placer des limites directes et indirectes sur la durée de vie du boson de Higgs.
Quelques centaines de bosons de Higgs produits dans ATLAS et CMS se sont désintégrés en deux photons $(\gamma \gamma)$. Dans ce mode de désintégration, le bruit de fond est abondant, mais l'excellente résolution en masse permet aux deux expériences de détecter un signal significatif (fig. 2).

En combinant les résultats de deux analyses portant sur les modes de désintégration en bb et $\tau \tau$, CMS obtient un fort indice du couplage du boson de Higgs aux fermions, et ATLAS rapporte une forte indication de désintégration du boson de Higgs en deux leptons $\tau$. En revanche, la désintégration en deux muons n'est pas détectée. Or le muon et le lepton $\tau$ sont des leptons qui ne different que par leurs masses (la masse du lepton $\tau$ est environ dix-sept fois la masse du muon). C'est un résultat important,
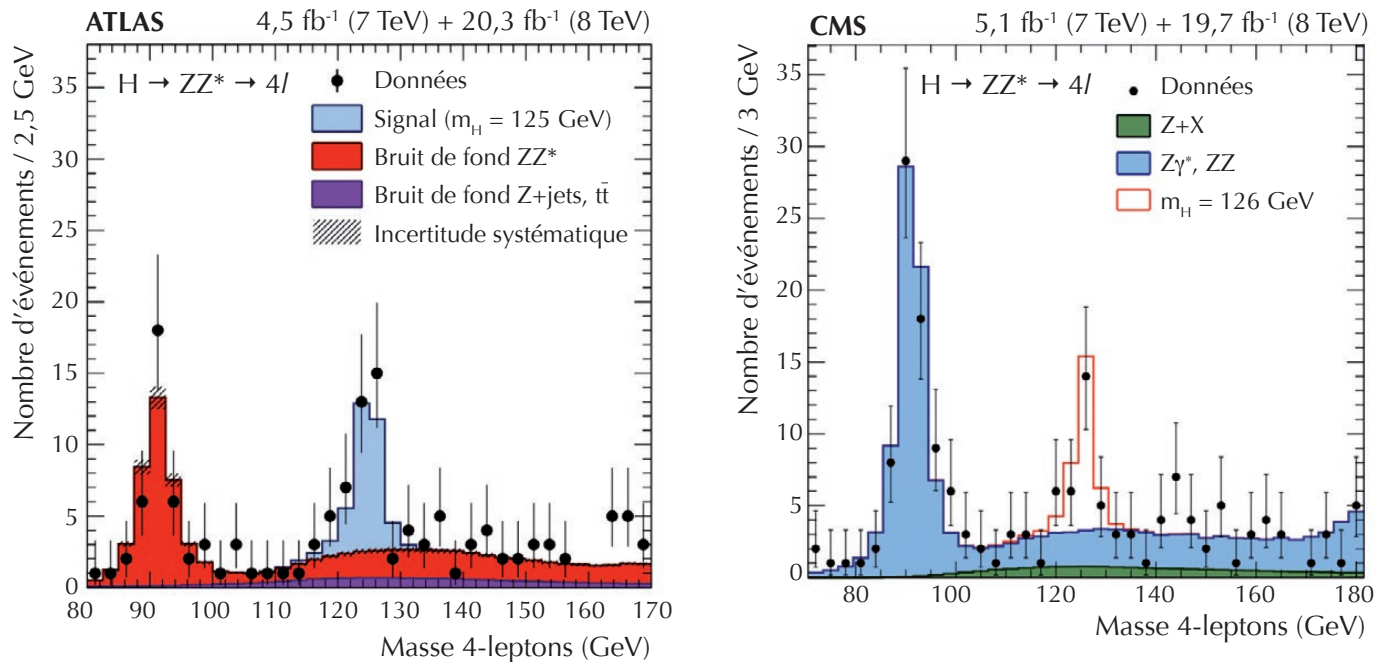

1. Signal du boson de Higgs dans sa désintégration $\mathbf{Z Z}$ : distribution de la masse combinée d'un système de quatre leptons entre 80 et $170 \mathrm{GeV}$ dans ATLAS (à gauche), et entre 70 et $180 \mathrm{GeV}$ dans CMS (à droite). Les pics autour de $91 \mathrm{GeV}$ et $125 \mathrm{GeV}$ sont respectivement les signaux des désintégrations du boson $Z$ et du boson de Higgs en quatre leptons. Les simulations, représentées par des histogrammes colorés, décrivent les données de façon satisfaisante.
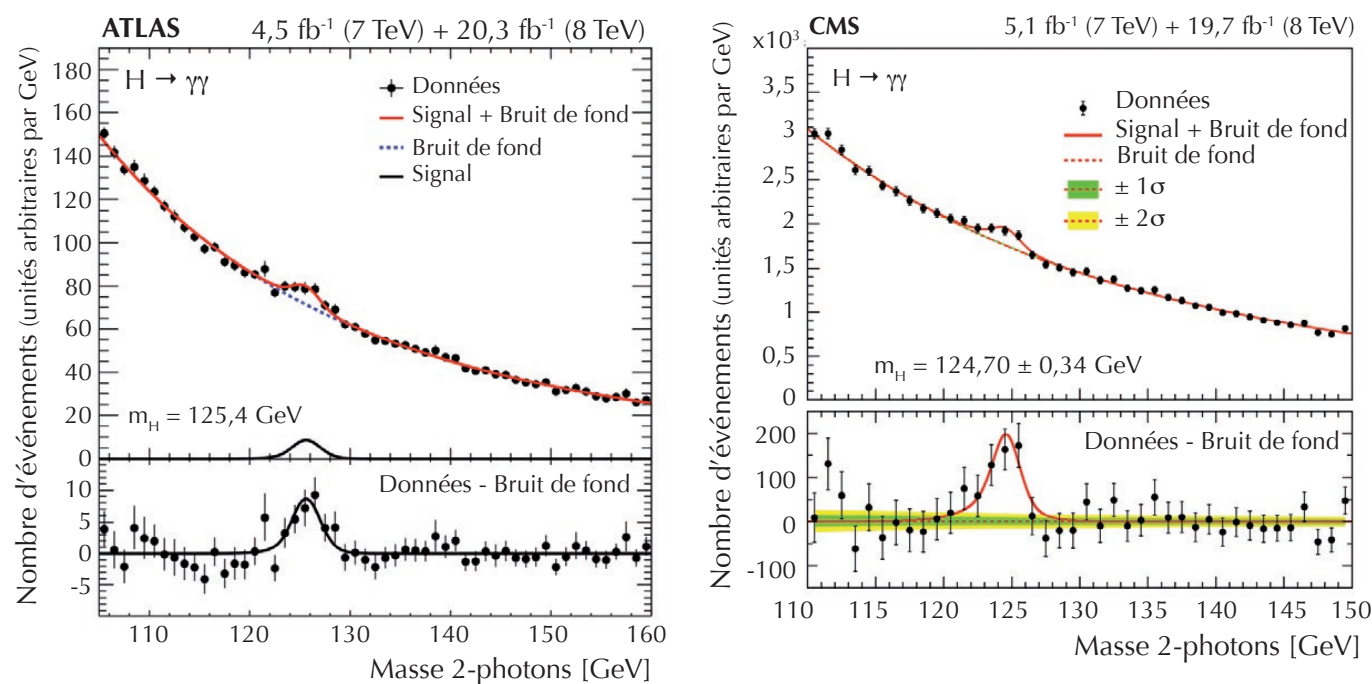

2. Signal du boson de Higgs dans le mode de désintégration $\gamma \gamma$ : distribution de la masse combinée d'un système de deux photons entre 100 et $160 \mathrm{GeV}$ dans ATLAS (à gauche) et entre 110 et $150 \mathrm{GeV}$ dans CMS (à droite). Les données sont représentées par des points noirs avec barres d'incertitude. Les courbes rouges représentent les ajustements des données. Les panneaux inférieurs présentent les données et les ajustements après soustraction de la fonction ajustée décrivant le bruit de fond. Le signal, clairement visible autour de $125 \mathrm{GeV}$, comprend plusieurs centaines de désintégrations du boson de Higgs en deux photons. 
$>>$

qui confirme que les couplages du boson de Higgs dépendent de la masse des particules. Compte-tenu du rapport des masses du muon et du lepton $\tau$, cent fois plus de données sont théoriquement nécessaires pour observer la désintégration du boson de Higgs en deux muons.

ATLAS et CMS mesurent la masse $m_{H}$ du boson de Higgs, séparément dans les modes $\gamma \gamma$ et ZZ. Ces quatre mesures, statistiquement indépendantes, sont en bon accord (fig. 3) et sont combinées pour une détermination de cette masse, $\mathrm{m}_{\mathrm{H}}=125,09 \pm 0,24 \mathrm{GeV}$, avec une incertitude relative de $0,2 \%$, qui en fait l'une des mesures de masse les plus précises obtenues au LHC à ce jour.

La figure 4 présente le résumé des mesures des taux de production du boson de Higgs par ATLAS et CMS dans les cinq principaux modes. La quantité portée en abscisse, qui est le signal observé rapporté à la prédiction théorique, vaut 1 dans l'hypothèse du MS avec le boson de Higgs à la masse de $125 \mathrm{GeV}$ et 0 en absence de boson de Higgs. On constate que toutes les mesures sont compatibles avec 1 et que les mesures dans les modes WW, ZZ et $\gamma \gamma$ excluent la valeur 0 avec un fort degré de confiance. Les valeurs combinées sont $1,18 \pm 0,15$ et $1,00 \pm 0,14$ pour ATLAS et CMS, respectivement. Les deux expériences observent la production et les désintégrations en accord avec la théorie avec une précision de l'ordre de 15\%, ce qui est remarquable compte tenu des difficultés expérimentales et de la complexité des calculs théoriques mis en jeu.

Les résultats peuvent être interprétés en termes de couplages du boson de Higgs aux particules élémentaires les plus massives (fig. 5). Le couplage au quark top intervient dans la production ggF et dans la désintégration en deux photons. Les couplages aux bosons de jauge interviennent dans les productions VBF et $\mathrm{VH}$ et dans les désintégrations WW, ZZ et $\gamma \gamma$. Les couplages aux fermions découlent des observations dans les modes bb et $\boldsymbol{\tau} \tau$, et des limites dans le mode $\mu \mu$. Les données confirment, dans les incertitudes expérimentales, que la nouvelle particule se couple aux autres particules en fonction de leur masse, comme prédit pour le boson de Higgs. Il faut maintenant vérifier que ses nombres quantiques sont conformes aux prédictions.

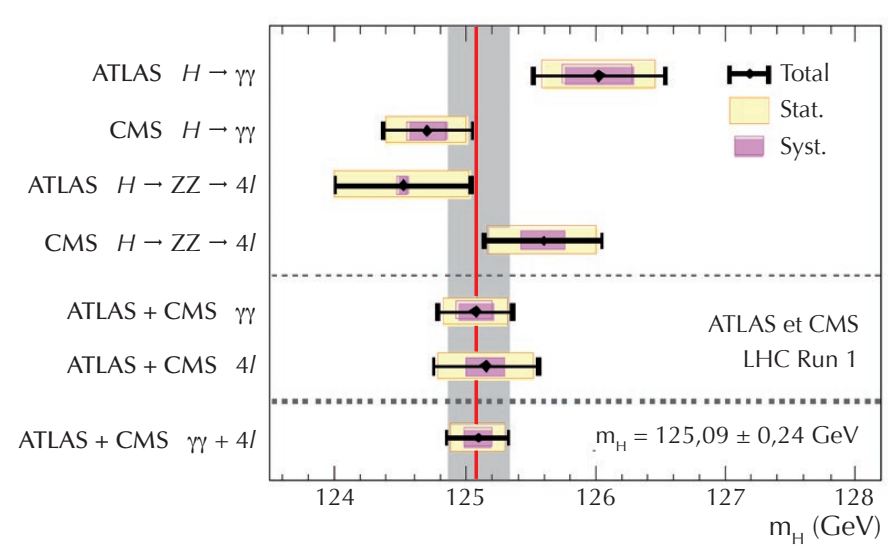

3. Mesures de la masse du boson de Higgs $\left(\mathrm{m}_{H}\right.$ en $\mathrm{GeV}$, en abscisse). Les quatre mesures indépendantes (quatre premières lignes) sont d'abord combinées par modes de désintégration du boson de Higgs (deux lignes suivantes). La mesure combinée finale (dernière ligne) est aussi représentée par une ligne verticale rouge à 125,09 GeV et son incertitude $( \pm 0,24 \mathrm{GeV})$ par une bande verticale grise.

\section{ATLAS}

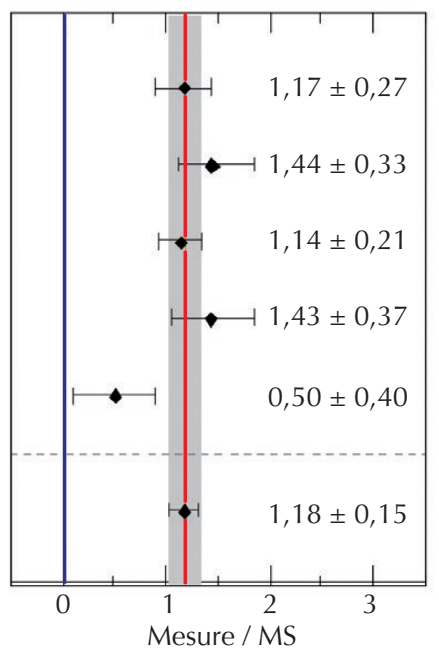

CMS

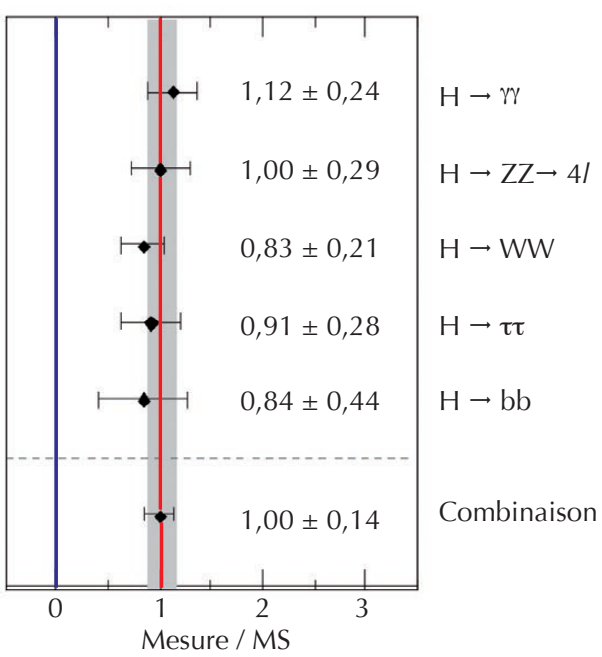

4. Résumé des mesures dans les cinq principaux modes de désintégration du boson de Higgs, pour ATLAS (à gauche) et pour CMS (à droite). L'axe des abscisses présente le taux de production du signal observé par rapport à celui du signal attendu dans l'hypothèse du boson de Higgs du MS. Les valeurs 1 et 0 correspondent au MS avec et sans boson de Higgs, respectivement. Toutes les mesures, représentées par des points avec barres d'erreur, sont compatibles avec la valeur 1 dans les incertitudes expérimentales. Celles-ci incluent une composante systématique, qui est dans tous les cas faible comparée à la composante statistique.

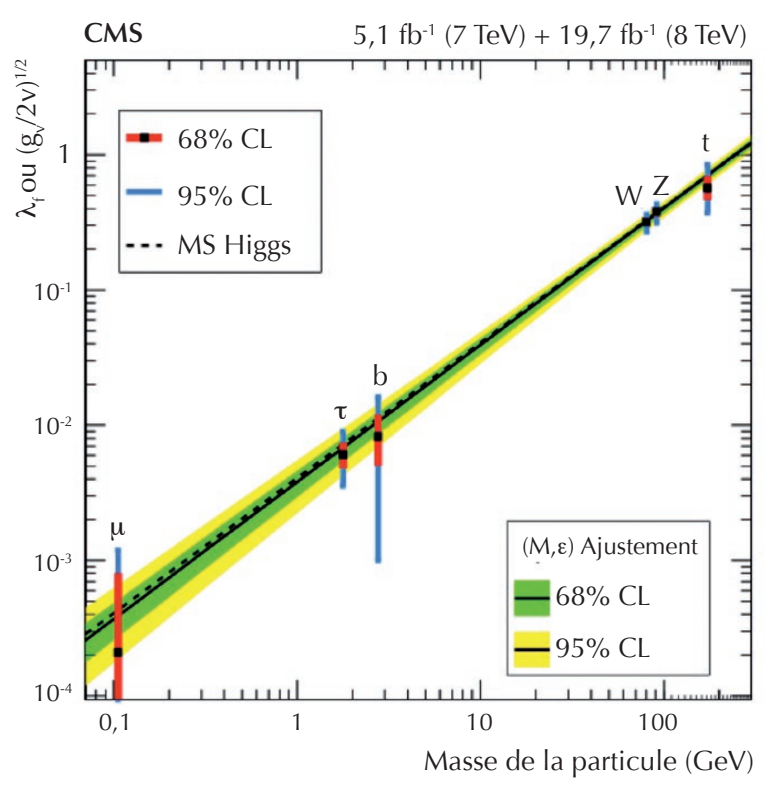

5. Vérification de la propriété fondamentale du boson de Higgs. Ici, les mesures de CMS sont interprétées en terme de couplages du boson de Higgs aux particules du MS : les bosons $W$ et $Z$, les quarks $t$ (top) et $b$, les leptons $\tau$ et $\mu$. Les couplages sont correctement normalisés entre fermions et bosons $\left(\lambda_{f}\right.$ représente le couplage des fermions et $(\mathrm{g} / 2 \mathrm{v})^{1 / 2}$ la racine carrée du couplage des bosons), et sont portés en fonction de la masse des particules considérées. Les échelles des deux axes sont logarithmiques. La relation de linéarité en fonction de la masse dans le MS est représentée par une ligne pointillée noire. La courbe continue noire représente l'ajustement linéaire des données, et les bandes vertes et jaunes les incertitudes à $68 \%$ et 95\% de degré de confiance (CL). 


\section{Mesure du spin et de la parité}

Comme dans tout système quantique, chaque particule possède des propriétés caractéristiques quantifiées, représentées par des nombres quantiques entiers ou demi-entiers. Par exemple le spin est une propriété intrinsèque, qui n'a pas d'équivalent classique, liée aux symétries d'espacetemps respectées par son champ. Une autre propriété intrinsèque d'une particule est la parité, qui est liée aux propriétés de symétrie de son champ sous une opération d'inversion des coordonnées d'espace. Le nombre quantique de parité, noté " $\mathrm{P}$ », peut prendre la valeur $\mathrm{P}=+1$ (parité positive) ou $\mathrm{P}=-1$ (parité négative).

Pour établir la nature de la particule découverte en 2012 par ATLAS et CMS, la détermination de ses nombres quantiques de spin $(\mathrm{J})$ et de parité $(\mathrm{P})$, notés collectivement " $\mathrm{J}^{\mathrm{P}}$ ", est cruciale. La spin-parité prédite pour le boson de Higgs dans le MS est $\mathrm{J}^{\mathrm{P}}=0^{+}$.

La conservation du moment cinétique angulaire dans les processus de désintégration (en particulier la désintégration $\gamma \gamma$ ), et les règles de somme de spins en mécanique quantique, indiquent que la nouvelle particule est un boson, c'est-à-dire une particule de spin entier.

Pour déterminer la valeur de $\mathrm{J}^{\mathrm{P}}$ de ce nouveau boson, les expériences ont analysé finement la cinématique des principaux modes de désintégration. Ainsi, en utilisant des fonctions de vraisemblances maximales, la probabilité pour que les distributions expérimentales observées soient compatibles avec celles attendues dans l'hypothèse que la particule découverte possède une spin-parité $\mathrm{J}^{\mathrm{P}}=0^{+}$, est comparée avec des hypothèses alternatives $\left(\mathrm{J}^{\mathrm{P}}=0^{-}, 1^{+}, 1^{-}, 2^{+}, 2^{-}\right)$.

Les fonctions de vraisemblances maximales sont construites à partir de variables discriminantes, telles que les variables liées à la direction d'émission des photons ou des leptons dans l'état final.

La compatibilité des données avec $1^{\prime}$ hypothèse $\mathrm{J}^{\mathrm{P}}=0^{+}$(par rapport à une hypothèse alternative considérée) est exprimée quantitativement par la valeur d'une quantité notée "CLs ", calculée à partir des fonctions de vraisemblances maximales. Ainsi une valeur faible de CLs, par exemple inférieure à $5 \%$, indique que l'hypothèse de $\mathrm{J}^{\mathrm{P}}$ alternative à celle du MS est rejetée avec un degré de confiance supérieur à $(1-\mathrm{CLs})=95 \%$.

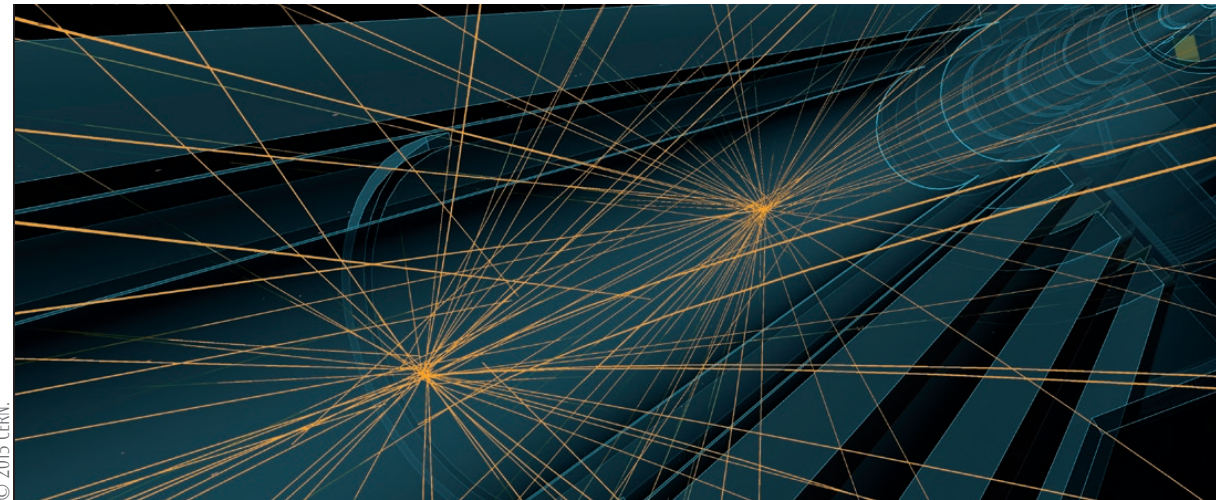

Double collision proton-proton à une énergie de 13 TeV, enregistrée par ATLAS le 21 mai 2015.
Les expériences considèrent plusieurs modèles alternatifs au MS, qui modifient les taux de désintégration et les modes de production de la nouvelle particule. Par exemple, le calcul du CLs est effectué pour différentes valeurs relatives des taux de production par interaction quark-antiquark et par interaction gluon-gluon.

La totalité des résultats obtenus par ATLAS et CMS est en accord avec l'hypothèse selon laquelle les nombres quantiques de spin et de parité de la nouvelle particule sont $\mathrm{J}^{\mathrm{P}}=0^{+}$, comme le prédit le MS. Les hypothèses alternatives sont toutes rejetées avec un degré de confiance supérieur à 95\%, et souvent à 99\%.

La figure 6 présente les degrés de confiance attendus et observés par ATLAS pour différentes hypothèses alternatives au MS.

$\gg>$

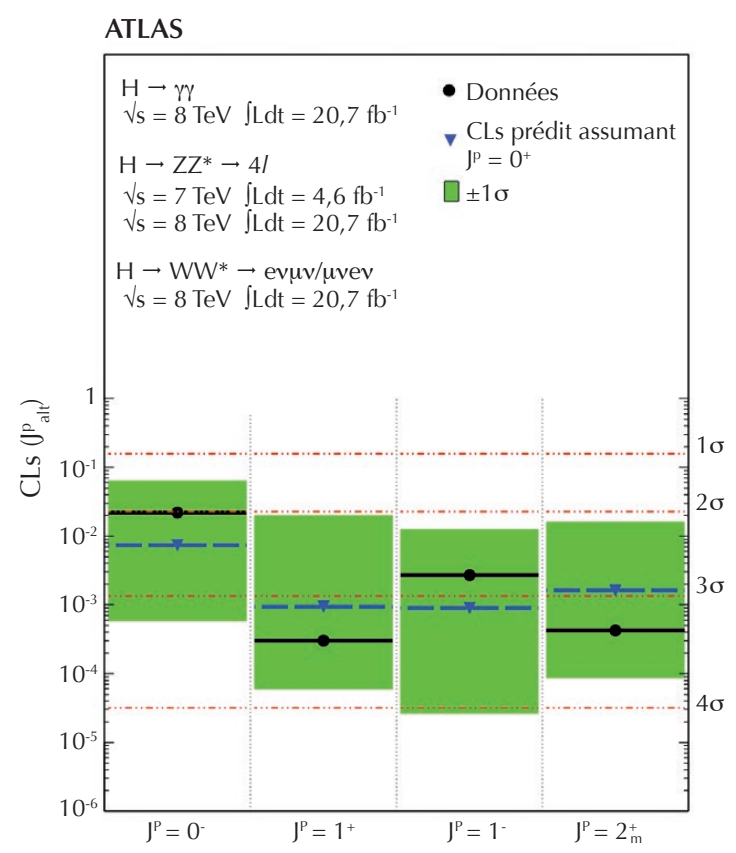

6. Spin (J) et parité (P) du boson de Higgs. Ici, les données d'ATLAS dans les trois modes WW, ZZ et $\gamma \gamma$ sont exploitées pour tester l'hypothèse des nombres quantiques prédits par le MS, $\mathrm{J}^{\mathrm{P}}=0^{+}$, face aux différentes alternatives $\mathrm{JP}_{\mathrm{alt}}=0^{-}, 1^{+}, 1^{-}$et $2^{+}$. Les tests d'hypothèse appliqués aux données démontrent quantitativement que les hypothèses alternatives sont fortement défavorisées par rapport à celle du MS. Une valeur donnée de CLs (JP alt), extraite des données (points noirs) par des méthodes statistiques et représentée sur l'axe vertical de gauche, indique que les hypothèses de spin et de parité alternative au MS, représentées sur l'axe horizontal, sont rejetées avec un niveau de confiance supérieur ou égal à 1-CLs. Les valeurs représentées par les triangles en bleu sont calculées en comparant des distributions simulées de variables caractéristiques (dans l'hypothèse que le spin et la parité du nouveau boson est $\mathrm{J}^{\mathrm{P}}=0^{+}$) avec des distributions simulées, où les valeurs de $\mathrm{J}^{\mathrm{P}}$ sont celles reportées en abscisse. Les bandes en vert montrent les valeurs du CLs (dans l'hypothèse $\mathrm{J}^{\mathrm{P}}=0^{+}$) qu'on obtiendrait dans 68\% des cas, simulées en comparant avec les valeurs alternatives de JP portées en abscisse. Les triangles bleus et les bandes vertes montrent que la simulation décrit les données. La figure soutient fortement l'hypothèse que les nombres quantiques du boson de Higgs sont ceux prédits par la théorie. 


\section{\〉}

\section{Le futur de la recherche dans le domaine du boson de Higgs}

Le spectre des particules prévues par le MS est désormais complet. Les propriétés de la particule découverte en 2012 par ATLAS et CMS correspondent à la dernière pièce manquante du MS, le boson de Higgs. C'est un succès indéniable du MS, qui confirme son extraordinaire pouvoir prédictif. Néanmoins, il y a de bonnes raisons de penser que le MS n'est pas une théorie complète. Parmi les nombreuses questions encore ouvertes, on peut citer, par exemple, celle de l'origine des masses extrêmement faibles mais non nulles des neutrinos (dont il semble qu'elle ne soit pas liée au mécanisme BEH), de la nature de la matière noire (postulée pour rendre compte d'observations astrophysiques et cosmologiques) et de l'excès de la matière sur l'antimatière dans l'Univers observable. Enfin, il faut mentionner le fait que le MS ne traite pas le problème de la gravitation quantique.

La découverte du boson de Higgs pose d'autres questions, d'ordre théorique. Selon le MS, la valeur théorique de la masse du boson de Higgs reçoit des corrections quantiques qui sont proportionnelles à l'échelle d'énergie jusqu'à laquelle la théorie est valable, par exemple celle de la grande unification des interactions électrofaible et nucléaire forte, qui est de nombreux ordres de grandeur supérieure à l'échelle des masses des bosons vecteurs $\mathrm{W}$ et $\mathrm{Z}$ et du boson de Higgs. Un ajustement fin (non naturel) des paramètres du MS dans la soustraction entre termes très grands est donc nécessaire pour obtenir in fine la valeur physique mesurée, $\mathrm{m}_{\mathrm{H}}=125 \mathrm{GeV}$. D'autre part, la valeur $125 \mathrm{GeV}$ de la masse est telle qu'il pourrait ne pas être nécessaire de faire intervenir de la nouvelle physique avant une énergie encore plus élevée, proche de l'énergie de Planck où la gravitation devient quantique.

Des théories alternatives et plusieurs extensions du MS évitent ces ajustements fins et tentent de répondre à certaines questions encore ouvertes. Une compréhension approfondie du mécanisme de $\mathrm{BEH}$ pourrait nous éclairer sur la voie à suivre pour dépasser le MS. Une liste non exhaustive des études à mener comprend les actions suivantes : compléter la mesure des propriétés du boson de Higgs (en particulier son couplage aux fermions, son autocouplage et son temps de vie), vérifier son rôle dans la diffusion des bosons $\mathrm{W}$ et $\mathrm{Z}$ à haute énergie, vérifier qu'il est une particule élémentaire et non composite, rechercher si d'autres bosons de Higgs existent.

\section{Conclusions}

Après un arrêt programmé de 18 mois, le collisionneur LHC a redémarré en juin 2015 tout d'abord à une énergie de $13 \mathrm{TeV}$ avant de se stabiliser à l'énergie nominale de $14 \mathrm{TeV}$, dans le but de sonder un domaine d'énergie inexploré, avec l'espoir d'observer de nouveaux phénomènes permettant de tracer la voie au-delà du MS. On prévoit de recueillir d'ici à 2022 dix fois plus de données que la quantité obtenue depuis 2010.

Les principaux objectifs de l'étude du boson de Higgs par ATLAS et CMS seront de confirmer sa désintégration en bb et $\tau \tau$ (pour mesurer précisément ses couplages avec les fermions) et de mettre en évidence sa production en association avec une paires de quarks top pour tester directement son couplage au quark top, la particule la plus massive du MS.

Les autres aspects du programme, qui comprennent les mesures de la diffusion des bosons $\mathrm{W}$ et $\mathrm{Z}$, du couplage du boson de Higgs au muon et de son autocouplage, nécessiteront une augmentation de la quantité de données par un facteur de l'ordre de 100. C'est l'objectif du programme High Luminosity LHC, qui devrait démarrer après 2025.

La recherche de bosons de Higgs additionnels, prédits dans certaines théories de nouvelle physique comme la supersymétrie, de la désintégration du boson de Higgs en particules hypothétiques de matière noire, ou l'étude de sa nature élémentaire ou composite, se poursuivront tout au long de ce programme expérimental.

D'autres mesures plus délicates encore, par exemple la mesure précise du temps de vie du boson de Higgs, nécessiteront probablement une nouvelle machine tel qu'un collisionneur électron-positon. Des projets très avancés sont en discussion, et l'un ou l'autre pourrait voir le jour dans un futur proche.

La connaissance des constituants ultimes de la matière et des forces fondamentales qui s'exercent entre eux avance à grand pas, mais la route est encore longue pour comprendre en profondeur les lois physiques qui régissent notre Univers.
[^] La portée d'une force dépend de la masse de la particule médiatrice. Déjà, dans les années 1930, le physicien japonais Hideki Yukawa, en s'appuyant sur le principe d'incertitude de Heisenberg, avait montré qu'une interaction entre particules était liée à l'échange d'un boson massif et que cet échange donnait lieu à une force de portée inversement proportionnelle à la masse du boson. Si le boson échangé est de masse nulle, la force est de portée infinie, comme c'est le cas de l'interaction électromagnétique avec le photon. De la portée de l'interaction nucléaire forte entre nucléons (protons et neutrons), Yukawa avait déduit l'existence de bosons de masse $0,1 \mathrm{GeV}$, identifiés aux pions découverts par la suite. Cette interaction de courte portée entre nucléons découle elle-même de l'interaction forte entre les quarks qui constituent les pions et les nucléons. Celle-ci, due à l'échange de médiateurs de masse nulle, les gluons, est pourtant de portée encore plus courte : ce paradoxe découle de la propriété de confinement des quarks, dont la conséquence est que ces derniers ne sont pas observables directement à l'état libre.

\section{Références}

1 • « Le Modèle Standard ", Élémentaire, n6 (2008) http://elementaire.web.lal.in2p3.fr/documents/ numero6/N6MS.pdf

2• L. di Ciaccio et G. Hamel de Monchenault, « Derniers résultats sur la recherche du boson de Higgs au LHC ", Reflets de la physique 28 (2012) 15-17.

3• L. di Ciaccio et G. Hamel de Monchenault, " Découverte du boson de Higgs au LHC », Reflets de la Physique 31 (2012) 17-19.

4 F. Malek, « Le calcul scientifique des expériences LHC - Une grille de production mondiale». Reflets de la physique, 20 (2010) 11.

5• Les publications d'ATLAS et CMS sur le boson de Higgs sont disponibles sur les sites suivants https://twiki.cern.ch/twiki/bin/view/AtlasPublic/ HiggsPublicResults, https://twiki.cern.ch/twiki/bin/view/CMSPublic/ PhysicsResultsHIG

6• G. Bernardi, « La recherche du boson de Higgs au Tevatron », Reflets de la physique, 14 (2009) 10.

\section{En savoir plus}

Gilles Cohen-Tannoudji et Michel Spiro, Le boson et le chapeau mexicain, Collection Folio essais ( $\left.n^{\circ} 579\right)$, Gallimard (2013).

Daniel Denegri, Claude Guyot, Andreas Hoecker et Lydia Roos, L'aventure du grand collisionneur LHC, EDP Sciences (2014). 